\title{
9 Orientierung im pluralistischen Ethikdiskurs
}

\author{
9.1 Deutungen des Theoriepluralismus \\ 9.2 Versuche der Problemvermeidung \\ 9.3 Unumgänglichkeit des Pluralismusproblems \\ 9.4 Differenzierung von Problemebenen \\ 9.5 Ausgangspunkte ethischer Orientierung
}

\section{1 | Deutungen des Theoriepluralismus}

Ernüchternde Ethik? Zu Beginn des 20. Jahrhunderts macht Harold A. Prichard eine Beobachtung, die sich auch heute bestätigen lässt. Das Studium der Moralphilosophie hinterlässt oft ein vages Gefühl der Unzufriedenheit:

"Probably to most students of Moral Philosophy there comes a time when they feel a vague sense of dissatisfaction with the whole subject."

Dafür mag zum Teil die für Einführungen übliche Darstellungsweise verantwortlich sein, die auch im vorangehenden Teil des vorliegenden Buchs gewählt wurde:

"A so-called textbook approach to moral theory presents several competing theories and then proceeds to criticize them. Often the criticisms are so severe that each theory seems fatally wounded, and readers become skeptical about the value of ethical theory in general."

Keine Darstellung kann allerdings Folgendes kaschieren: In der Geschichte der Moralphilosophie sind teilweise unterschiedliche Antworten auf die Frage gegeben worden, an welchen Prinzipien wir uns in letzter Hinsicht orientieren sollen und warum. Manche dieser Diskussionen dauern noch an. Auch am gegenwärtigen Diskurs beteiligen sich beispielsweise neo-aristotelische oder sentimentalistisch orientierte Tugendethiker/innen, Utilitarist/innen, Kantianer/innen, Vertragstheoretiker/innen und Vertreter/innen sgemischter Theorien, die Elemente aus verschiedenen Traditionen auf unterschiedliche Weise kombinieren. Auch metaethische Grundlagendiskurse sind noch nicht abgeschlossen.

Deutungsmöglichkeiten: Dieser Befund lässt sich allerdings selbst unterschiedlich interpretieren. Eine radikal pessimistische Deutung läge in der Auffassung, dass die Moralphilosophie die in sie gesetzte Hoffnung, angesichts moralischer Alltagskontroversen die richtigen Werte und Normen dingfest machen zu können, schlichtweg nicht erfüllt hat. Ihr Beitrag zu unserem praktischen Orientierungsvermögen sei bestenfalls wertlos. Schlimmstenfalls habe sie zusätzliche Verunsicherung ge-
Prichard 1912, S. 21

Beauchamp/ Childress 2013, S. 351

Aktueller Theoriepluralismus 
Moralische Fortschritte schaffen, indem sie den moralischen Alltagsdisputen weitere Theoriekontroversen hinzugefügt hat. Optimistischere Deutungen können an verschiedenen Punkten ansetzen. Erstens sind nicht alle Unterschiede zwischen moralphilosophischen Theorien mit Dissensen bezüglich der Gültigkeit moralischer Urteile verbunden. Teils resultieren sie vielmehr aus unterschiedlichen Fragestellungen, Situationsdeutungen oder strittigen nicht-moralischen Annahmen. Nahezu alle normativen Ethiken sprechen ja der Befriedigung von Bedürfnissen oder Interessen oder der Realisierung individueller oder gemeinschaftlicher Werte moralische Bedeutung zu. Soweit sie sich nicht mit der Aufstellung abstrakter Prinzipien begnügen, sondern auch situationsspezifische Orientierung geben wollen, müssen sie deshalb stets auf Annahmen Bezug nehmen, die Gegenstand »vernünftiger Meinungsverschiedenheiten« (Rawls 1993, S. 54 ff.; McMahon 2009) und/oder der freien Selbstbestimmung der Betroffenen sind. Zweitens sind keineswegs alle moralischen Überzeugungen, ethiktheoretischen Annahmen, Argumente und Methoden gleichermaßen kontrovers. Neben moralischen Dissensen und Theoriekontroversen lassen sich auch Konsensbereiche und Konvergenzen feststellen (s. u.). Auch scheint es, dass die moralphilosophische Debatte durchaus Entwicklungen befördert hat, die weithin - vielfach auch theorieübergreifend - als moralische Fortschritte anerkannt sind. Das gilt vor allem für die Durchsetzung universeller Menschenrechte. Ehemals einflussreiche (Schein-) Argumente für die Aufrechterhaltung der Sklaverei oder gegen das Frauenwahlrecht gelten allgemein als widerlegt. Ihre theoretische Widerlegung war und ist für die praktische Zurückdrängung dieser Diskriminierungen durchaus von Bedeutung.

\section{2 | Versuche der Problemvermeidung}

Probleme des Moralskeptizismus
Skeptizismus oder provisorische Moral: Wer die pessimistische Deutung akzeptiert, könnte zu der Annahme neigen, dass moralische Urteile entweder gar keinen Wahrheitswert haben (Nonkognitivismus), keiner rationalen Begründung zugänglich (Skeptizismus), sämtlich fehlerhaft (Irrtumstheorie) oder stets nur im Rahmen bestimmter Urteilskontexte wahr sind (Relativismus). Die Diskussion im vorangehenden Kapitel hat gezeigt, dass all diese Positionen selbst schwerwiegenden Einwänden ausgesetzt sind (siehe Kap. 8). Eventuell ließe sich eine schwächere Form des Moralskeptizismus denken, die lediglich behauptet, bisher sei noch gar kein moralisches Urteil und keine Norm hinreichend begründet worden. Um diese Position zu verteidigen, muss man allerdings einen Standard hinreichender Begründung definieren und überdies zeigen, dass wir uns ausschließlich an moralischen Urteilen und Normen orientieren sollten, die diesem Begründungsstandard genügen. Zumindest diese `Metanorm (»Orientiere Dich nur an Normen, die dem Begründungsstandard xy genügen!«) müsste dann den moralskeptischen Zweifeln standhalten. Es wäre dann zu zeigen, warum wir diese Metanorm eher akzeptieren sollten, als eine, die weniger strenge Begründungsanforderungen vorsieht: 
Warum sollten wir sie beispielsweise den Maximen einer provisorischen Moral vorziehen, die bei Entscheidungen unter Handlungsdruck und normativer Ungewissheit eine Orientierung an den jeweils besten verfügbaren Gründen empfiehlt?

\section{Descartes' provisorische Moral}

Als rationalistischer Philosoph der frühen Neuzeit sucht René Descartes nach einer Basis sicherer Erkenntnis, die auch noch dem radikalsten Zweifel standhält. Da wir jedoch auch in Situationen handeln müssen, in denen eine solche Basis (noch) nicht gelegt ist, schlägt er im Discours de la méthode (1637) Maximen einer provisorischen Moral vor. Erstens solle man im Zweifelsfall den Sitten besonnener Personen im eigenen Lebensumfeld folgen und dabei, wenn man »unter mehreren Überzeugungen, die gleichermaßen anerkannt werden" (Descartes 1997, S. 39 [1637, S. 25]) wählen muss, die jeweils moderateren wählen, weil diese im Fall eines Fehlgriffs weniger weit vom richtigen Weg entfernt sind als die extremen. Zweitens sollten wir, "wenn es nicht in unserer Macht steht, die wahrsten Ansichten zu erkennen, den wahrscheinlichsten folgen « (ebd., S. 40 [26]). Dies sollten wir dann auch mit der nötigen Entschlossenheit und ohne Gewissensbisse tun, da wir sonst in keinem Fall etwas Vernünftiges zuwege bringen werden. Drittens sollten wir eher versuchen, unsere Bedürfnisse an die Umstände anzupassen als umgekehrt und viertens uns nach Kräften bemühen, Verstand und praktische Urteilskraft weiterzuentwickeln (zu Descartes' Ethik vgl. Perler 1998, insbes. S. 231 ff.; Marshall 2018; zum Konzept provisorischer Moral Hubig/Luckner 2013; Hubig 2007).

Anti-Theorie: Mitunter verbindet sich die pessimistische Deutung der Orientierungsleistungen ethischer Theorien auch mit der generellen Abwehr theoretischer Problematisierungen und der Beschwörung des (vermeintlich) vortheoretisch Unstrittigen: Sind wir überhaupt ernsthaft im Zweifel darüber, was ein gutes Leben ausmacht oder was wir einander schulden? Oder 'wissen «ir dies nicht sim Grunde unseres Herzens` recht genau? Sind nicht vielleicht die Zweifel selbst - oder möglicherweise schon das Streben nach sicherem und explizitem Wissen - das Problem? In diesem Sinne hat sogar Jürgen Habermas einmal formuliert:

"Die moralischen Alltagsintuitionen bedürfen der Aufklärung des Philosophen nicht. [...] Die philosophische Ethik hat eine aufklärende Funktion allenfalls gegenüber den Verwirrungen, die sie selbst im Bewußtsein der Gebildeten angerichtet hat."

Während es sich bei diesem Zitat um eine vereinzelte Äußerung handelt, die mit Habermas' aufklärerischem Projekt einer »Rationalisierung der Lebenswelt« (Habermas 1981) und den Grundideen seiner Diskurstheorie von Recht und Moral (Habermas 1983, 1991, 1992, 1996) kaum vereinbar erscheint, basieren »anti-theoretische« Positionen auf genau solchen Annahmen (zur Übersicht vgl. Clarke/Simpson 1989). Sie haben durchaus 
Probleme der 'Anti-Theories

Ricken 2013, einen Anschein von Plausibilität. »Wer erst ein Prinzip hervorkramen muss, um Gut und Böse zu unterscheiden, ist ein Schuft", meint schon der niederländische Schriftsteller Multatuli (1877, Bd. IV, S. 127; Übersetzung MHW). Anti-Theoretiker/innen versuchen daher, lebensweltliche Auffassungen vom Guten, konkrete moralische Handlungsorientierungen und Intuitionen vor den Abstraktionen und (vermeintlichen) Verzerrungen zu bewahren, die sich beim Versuch ihrer theoretischen Rekonstruktion ergeben.

Anti-theoretische Theorie? Wachsamkeit gegenüber möglichen Verzerrungen bei der theoretischen Rekonstruktion moralischer Phänomene ist sicher wichtig. Als moralphilosophische Grundposition sieht sich die Anti-Theorie jedoch gravierenden Einwänden ausgesetzt: Erstens sind schon moralische Alltagsüberzeugungen und -praktiken von Traditionen ethischen >Theoretisierens` geprägt und zum Teil selbst an rabstrakten Prinzipien orientiert (man denke an die Goldene Regel). Wie schon eingangs erwähnt gehen ethische Theoriebildung und moralische Alltagsüberlegungen ineinander über (vgl. auch Birnbacher 2013, S. 114 f.) und unterscheiden sich vor allem durch die Strenge der Explikationsansprüche:

\section{"Vorphilosophisches moralisches Bewusstsein und Ethik sind in dem Sinn nicht zu trennen, dass das moralische Bewusstsein immer schon nach einer Begründung fragt."}

Zweitens können Muster prinzipiengeleiteten Handelns durch wiederholtes Einüben so internalisiert werden, dass die explizite Bezugnahme auf ein moralisches Prinzip in Standardsituationen überflüssig ist. Multatulis Urteil muss also gar nicht als `anti-theoretische` Stellungnahme verstanden werden. Sie lässt sich auch als Kritik an Personen lesen, deren moralische Bildung so oberflächlich geblieben ist, dass sie sich auch in moralisch eindeutigen Situationen noch durch den expliziten Rückgriff auf Prinzipien rückversichern müssen - ebenso wie jemand, der eine Sprache noch nicht sicher beherrscht, sich häufiger die Regeln der Grammatik bewusst machen muss. Drittens erscheint die Behauptung, es gebe auf vor-theoretischer Ebene keine wesentlichen praktischen Unsicherheiten oder Kontroversen, höchst unplausibel, wenn man den historischen Wandel moralischer Überzeugungen oder auch aktuelle politisch-ethische Debatten betrachtet. Viertens sieht sich der Versuch, die >vortheoretischer moralische Wirklichkeit ganz generell gegen Theoriebildung auszuspielen, der Schwierigkeit ausgesetzt, jene moralische Wirklichkeit zunächst einmal so einzugrenzen, dass sich überhaupt auf sie verweisen lässt. Diese Eingrenzung ist wiederum nur durch rabstrakte begriffliche Kennzeichnung möglich. Deshalb ist fraglich, ob sich eine Anti-Theorie überhaupt widerspruchsfrei formulieren lässt (Fotion 2014). Denn sobald Anti-Theoretiker/innen in systematischer Weise dafür argumentieren wollen, dass es prinzipiell besser ist, ethische Theoriebildung zugunsten einer Orientierung der Art $x$ (hier wäre irgendeine Beschreibung von $x$ oder ein begrifflicher Verweis auf $x$ nötig) zu unterlassen, vertreten sie nolens volens selbst eine bestimmte ethische Theorie. 
Common morality: Ähnliche Überlegungen treffen auch auf common morality Ansätze zu. So ist Bernard Gert (1998) darin zuzustimmen, dass wir uns bemühen sollten, die Strukturen unserer vortheoretischen Moralpraxis in ihrer vollen Komplexität zu erfassen, statt ihr mit groben Prinzipien zu Leibe zu rücken, die wir durch die Abstraktion eines einseitigen Zerrbildes dieser Moralpraxis gewonnen haben. Es ist jedoch nicht möglich, sich sozusagen im direkten Zugriff des Wesenskerns der vortheoretischen Moralpraxis zu bemächtigen, ohne dabei selbst begrifflich-theoretische Annahmen zu investieren. Den Anspruch, das Wesentliche der vortheoretischen Moral adäquat nachzukonstruieren, haben Hume und Kant ebenso erhoben wie die Vertreter/innen der common morality Ansätze. Auch stehen diesen keine grundsätzlich anderen $\mathrm{Me}$ thoden als jenen zur Verfügung, um (wie Kant dies nennt) den »Übergang von der populären sittlichen Weltweisheit zur Metaphysik der Sitten « (GMS, 2. Abschnitt) zu bewältigen. Wir können eine vortheoretische Moralpraxis und deren moralphilosophische Rekonstruktion nämlich nicht einfach wie Original und Abbild nebeneinanderstellen, um die Korrektheit des Abbildes zu beurteilen. Denn schon die Entscheidung darüber, was überhaupt zur 'geteilten Moralität` gehört, ist theoretisch voraussetzungsvoll. Wir brauchen zunächst ein Vorverständnis dessen, wonach wir suchen, damit wir mit der Suche überhaupt beginnen können. (Genau aus diesem Grund wirkt es komisch, wenn sich Pippi Langstrumpf auf die Suche nach einem "Spunk« macht, ohne ein Vorverständnis davon, was das von ihr frei erfundene Wort »Spunk« bedeutet). Das Vorverständnis sollte offenbar möglichst unkontrovers sein. Wie eingangs erwähnt, enthält Gerts Moraldefinition jedoch Festlegungen, die in der ethischen Diskussion durchaus strittig sind. Ferner wäre es mit dem Nachweis, dass die eigene Moraltheorie ein adäquates Bild der vortheoretischen Moralpraxis zeichnet, noch nicht getan. Es sollte auch gezeigt werden, dass diese Praxis vernünftig ist (so auch Gert 1998, S. 3 6, 17). Festzuhalten ist daher, dass es sich beim common morality Ansatz ebenso wie bei den Theorien Kants oder Humes, dem Utilitarismus etc. um eine bestimmte normativ-ethische Theorie handelt, die ein spezifisches Verständnis der Moral rational zu begründen versucht.

Principlism: Das zuletzt Gesagte trifft auch auf den dominierenden Ansatz der Medizinethik zu, der unter dem Namen Principlism bekannt ist (Beauchamp/Childress 2013). Im Deutschen wird häufig der Name "Prinzipienethik « für diesen Ansatz gebraucht. Dieser Begriff ist allerdings mehrdeutig, weil er ebenso für Theorien wie den Utilitarismus oder die Ethik Kants verwendet wird, die ein einziges `höchstes` Moralprinzip vorschlagen (siehe Kap. 5.1). Die Pointe des von Tom L. Beauchamp und James F. Childress vertretenen Principlism besteht hingegen gerade darin, kein derartiges oberstes Prinzip vorzusehen, sondern nur vier >mittlere Prinzipien (autonomy, nonmaleficience, beneficience, justice). Diese Prinzipien sollen gegeneinander abgewogen werden, ohne dass wir uns bei der Abwägung an einem höherrangigen Prinzip orientieren könnten. Auch die Vertreter/innen des Principlism halten Ansätze wie den Utilitarismus, Kantianismus oder Kontraktualismus für unbefriedigend und auch sie argumentieren, dass in diesen Theorien jeweils einzelne Ele- 
mente der Moral überbetont und andere vernachlässigt werden. In jüngerer Zeit beziehen sie sich zudem auch explizit - wenngleich mit gewissen Vorbehalten - auf den common morality Ansatz. Nun mag man die Befürchtung hegen, dass der Verzicht des Principlism auf ein höchstes Moralprinzip oder Abwägungskriterium zu Desorientierung oder moralischer Beliebigkeit führt oder wohlfeile Pseudo-Rechtfertigungen für die jeweiligen Wunschlösungen ermöglicht. Oder man mag argumentieren, dass der Principlism gut geeignet ist, das Spektrum moralisch relevanter Aspekte unverkürzt wahrzunehmen, moralische Dissense zu lokalisieren und damit die Diskussion voranzubringen. Problematisch aber wäre die Annahme, dass der Principlism sich ganz grundsätzlich von anderen Ansätzen normativer Ethik unterscheidet. Beauchamps und Childress' eigene Ausführungen bleiben in dieser Hinsicht unklar (vgl. Beauchamp/ Childress 2013, Kap. 9 und 10). So distanzieren sie sich von anti-theoretischen Ansätzen, wollen ihren Ansatz jedoch auch nicht als >Theorier bezeichnen. Dabei legen sie allerdings so strenge Kriterien an die Verwendung des Begriffs >Theorie an, dass er auch auf andere traditionelle Ansätze normativer Ethik nicht mehr passt. Sicherlich lässt der Principlism durch den Verzicht auf ein höchstes Moralprinzip der Abwägung mehr Raum als manch anderer Ansatz. Die Rolle moralischer Urteilskraft hat indes schon Aristoteles betont, und auch David Ross vertritt ausdrücklich eine Form von Pflichtenpluralismus, der es nötig macht, qualitativ unterschiedliche »Prima-facie-Pflichten « gegeneinander abzuwägen (Ross 2009; siehe Kap. 5.2.3). Auch die zentrale Rolle, die der Principlism der Methode des Überlegungsgleichgewichts beimisst, ist alles andere als ein Alleinstellungsmerkmal. Versteht man unter einer ethischen Theorie ganz allgemein eine mehr oder weniger umfangreiche und systematische Verbindung aus moralischen Urteilen oder Normen mit Argumenten für diese Urteile oder Normen, so ist auch der Principlism nichts anderes als eine bestimmte ethische Theorie und keineswegs eine Alternative zur ethischen Theoriebildung als solcher.

\section{3 | Unumgänglichkeit des Pluralismusproblems}

Bescheidenheit und Dogmatismus: Die Einsicht, dass common morality Ansätze, Principlism und andere Theorien, die sich auf die Methode des Überlegungsgleichgewichts stützen, nichts grundsätzlich anderes sind als andere normativ-ethische Theorien, macht auf einen wichtigen Umstand aufmerksam. In seiner Schrift Über die vierfache Wurzel des Satzes vom zureichenden Grunde (1813/1847) hält Schopenhauer fest, »[d]as Gesetz der Kausalität« sei "nicht so gefällig, sich brauchen zu lassen, wie ein Fiaker, den man, angekommen wo man hingewollt, nach Hause schickt. Vielmehr gleicht es dem, von Goethes Zauberlehrlinge belebten Besen, der, ein Mal in Aktivität gesetzt, gar nicht wieder aufhört zu laufen und zu schöpfen" (Schopenhauer 1988, Bd. 1, S. 38). Max Weber hat Schopenhauers Charakterisierung auf die Ethik der Bergpredigt umgemünzt (Weber 1988, S.438). Tatsächlich liegt hier jedoch ein Merkmal jedes Be- 
gründungs- oder Rechtfertigungsbemühens, einschließlich jeder ethischen Argumentation: Beginnt man einmal, Gründe anzuführen (etwa, wenn man eine bestimmte Handlungsweise $\mathrm{zu}$ rechtfertigen versucht, indem man sie aus einem bestimmten Prinzip ableitet), lädt man zu Anschlussfragen ein (etwa der Frage nach der Rechtfertigung des in Anspruch genommenen Prinzips). Dabei gilt einerseits, dass sich das Begründungsspiel nicht beliebig lange treiben lässt. Andererseits gilt jedoch, dass sich auch der Abbruch eines Begründungsbemühens grundsätzlich immer kritisch hinterfragen lässt: Ob wir ein höchstes Moralkriterium brauchen, wie etwa John St. Mill annimmt (Mill 1963 ff., Bd. 10, S. 205226), oder ob wir uns mit >mittleren Prinzipien begnügen sollten, wie Beauchamp und Childress meinen (s. o.), und wie die in Anspruch genommenen Prinzipien jeweils angewandt, gegeneinander abgewogen oder gerechtfertigt werden müssen, lässt sich wiederum nur mit ethischen Argumenten begründen - oder es bleibt eben nicht mehr als eine willkürliche Setzung. Hinter der Tugend der Bescheidenheit, die auf überzogenes Theoretisieren verzichten oder der Weisheit unserer vortheoretischen moralischen Intuitionen, Haltungen oder Institutionen vertrauen will, lauert stets die Gefahr des Dogmatismus, der blind auf ungeprüften Überzeugungen beharrt.

Theorieoffenheit moralischer Alltagsdebatten: Diese Einsicht lässt sich noch radikalisieren durch den schon mehrfach erwähnten Umstand, dass der Übergang zwischen moralischen Alltagsdiskussionen und dem moralphilosophischen Fachdiskurs fließend ist. Wer immer im Alltag eine Frage der Richtigkeit oder Legitimität aufwirft, betritt einen Raum normativer Gründe, der gewissermaßen in viele Richtungen geöffnet ist und sich nicht ohne Willkürgefahr gegen allgemeinere Prinzipienfragen, normativ-ethische Theoriefragen oder sogar metaethische Einwürfe abriegeln lässt. Das bedeutet keineswegs, dass es in jeder Alltagsdiskussion angemessen wäre, solche abstrakten Fragen aufzuwerfen. Wohl aber bedeutet es, dass wir auch in moralischen Alltagsdiskussionen sozusagen argumentativen Kredit aufnehmen, der sich unter geeigneten Umständen einfordern lässt. Dies könnte folgende Anschlussfrage provozieren: Wenn es stimmt, dass Personen, die in einen moralischen Alltagsdiskurs eintreten, etwa indem sie die Fairness einer Handlung, Norm oder Institution kritisieren oder verteidigen, sich dadurch Einwänden oder kritischen Nachfragen aussetzen - können sie es dann nicht einfach vermeiden und sich auf entsprechende Diskussionen gar nicht erst einlassen? In gewisser Weise können sie dies zweifellos. Faktisch ist es offenbar möglich, Diskussionen über Fragen der Handlungsorientierung abzubrechen oder ihnen von vornherein auszuweichen. In manchen Situationen kann dies auch legitim oder sogar geboten sein - z. B. wenn es dringlicher ist, jemanden aus einem brennenden Haus zu retten als ein moralphilosophisches Seminar fortzusetzen. Wer moralische Diskussionen vermeidet, ist dadurch jedoch nicht davor geschützt, von denjenigen kritisiert zu werden, die entsprechende Diskussionen führen (vgl. Darwall 2006, S. 262 ff.; Fn. 26).

Moralische Sanktionen: Mehr noch: Die Person ist auch nicht davor geschützt, dass andere sie ihrerseits zur Rede stellen oder ihr gar Sanktio- 
nen für Verhaltensweisen auferlegen, die sie für moralisch inakzeptabel halten. Anfechten kann sie die Legitimität dieser Sanktionen wiederum nur, indem sie mit relevanten Argumenten zu dieser normativen Frage Stellung nimmt. Das Schweigen einer radikalen Moralskeptikerin oder eines radikalen Moralskeptikers liefert ja kein Argument gegen die $\mathrm{Zu}$ lässigkeit von Sanktionen oder Zwangsmaßnahmen. Inwieweit Prinzipien des Zusammenlebens tatsächlich moralisch begründbar und die (wirklich oder vorgeblich) auf diese Prinzipien gestützten Sanktionen und Zwangsmaßnahmen moralisch legitim sind, lässt sich nicht anders als durch die Prüfung ethischer Argumente, Positionen und Theorien überprüfen.

\section{4 | Differenzierung von Problemebenen}

Theorie- und Methodenwahl: Damit sind wir gewissermaßen wieder beim Ausgangspunkt angelangt. Ethik soll auf vernünftige Weise klären, an welchen Prinzipien wir unser Leben, unser Handeln, unsere Handlungsbeurteilungen und unsere Institutionen orientieren sollen. Einige klassische Versuche, solche Prinzipien zu formulieren und zu begründen, wurden im zweiten Teil des Lehrbuchs vorgestellt. Dabei sind deutliche Unterschiede zwischen diesen Versuchen erkennbar geworden. Diese Erkenntnis hat zu der Frage geführt, inwieweit Ethik die in sie gesetzte Hoffnung, in praktischen Kontroversen Orientierung zu bieten, letztlich erfüllen kann. Denn soweit verschiedene ethische Theorien unterschiedliche Prinzipien vorschlagen, lässt sich praktische Orientierung nur gewinnen, wenn auch die Frage der Theorie- und Methodenwahl vernünftig zu klären ist. In den vorangehenden Abschnitten wurde erstens argumentiert, dass auch santi-theoretische` oder theorieskeptische Ansätze wie der Principlism oder der common morality Ansatz uns nicht von der Notwendigkeit der Theorie- und Methodenwahl entlasten können. Bestenfalls lassen sie sich selbst als eine bestimmte Art ethischer Theorien verstehen, für deren Plausibilität sich passende Argumente vorbringen lassen. Schlimmstenfalls handelt es sich um inkonsistente oder dogmatische Positionen (Düwell 2008, S. 89-95). Zweitens wurde argumentiert, dass ethiktheoretische Debatten sich natürlicherweise aus Versuchen ergeben, Lebensorientierungen, Handlungen oder Institutionen vernünftig zu begründen oder zu kritisieren.

Das Problem der Theorie- und Methodenwahl erscheint damit als unabweisbar, aber weiterhin ungelöst. Mit besonderer Dringlichkeit stellt es sich, wo konkrete Entscheidungen getroffen, vorbereitet oder reguliert werden sollen, zugleich aber höhere Erwartungen an die Explizitheit und Nachvollziehbarkeit von Begründungen gestellt werden, als dies in moralischen Alltagsdiskussionen der Fall ist. Das gilt vor allem für die bereichsspezifische Ethik und insbesondere für die ethische Beratung. Wie lässt sich mit diesem Problem angemessen umgehen? Zunächst ist zwischen drei Fragen zu unterscheiden:

Fragestellungen zur Methodenwahl
1. Wie kann man sich zwischen ethischen Überzeugungen, Theorien und Methoden überhaupt vernünftig entscheiden? 
2. Wie kann man mit verbleibenden Ungewissheiten hinsichtlich der Haltbarkeit ethischer Prinzipien oder ethisch relevanter empirischer Annahmen vernünftig umgehen?

3. Wie kann man mit faktischem Dissens über ethische Normen vernünftig umgehen?

Diese Fragen sind nicht unabhängig voneinander zu beantworten, aber doch wesentlich verschieden. Fragen des ersten Typs stellen sich, solange wir im Hinblick auf die Haltbarkeit ethischer Annahmen oder Ansätze unsicher sind, uns aber weiter bemühen, diesbezüglich zu einer korrekten Position zu kommen. Fragen des zweiten Typs stellen sich in Kontexten, in denen wir es für nicht aussichtsreich oder nicht verantwortbar halten, ethische (oder ethisch relevante empirische) Ungewissheiten vollständig auszuräumen. In solchen Kontexten suchen wir vernünftige höherstufige Prinzipien für den Umgang mit Ungewissheiten. Beispiele für solche Prinzipien sind Descartes' bereits erwähnte Maximen einer provisorischen Moral, Pascals »Wette«, das Vorsorgeprinzip oder Kalküle der Nutzen-Risiko-Abwägung. Für die höherstufigen Prinzipien selbst stellen sich dann grundsätzlich wieder Fragen des ersten Typs. Fragen des dritten Typs können sich sowohl aus der Perspektive von Personen stellen, die in Bezug auf die Richtigkeit bestimmter ethischer Positionen unsicher sind, als auch aus der Perspektive von Personen, die keine Zweifel an der Richtigkeit dieser Positionen haben. Auch für Personen, die von der ethischen Rechtfertigbarkeit bestimmter Normen überzeugt sind, ergeben sich vielfach praktische Probleme, wenn andere Personen im Handlungsumfeld diese Normen nicht anerkennen. Das Spektrum möglicher Reaktionen ist weit: Sie reichen vom Versuch, andere von der eigenen Auffassung zu überzeugen (wobei sich Fragen von Typ 1 stellen) über den Versuch, bestimmte Dissense einzuklammern und sich auf einen basalen modus vivendi zu einigen (wobei sich Fragen von Typ 2 stellen) bis zu Formen der Kompromissfindung, Toleranz oder Duldung, zu Sanktionen oder strategischem Verhalten, die ihrerseits Gegenstand ethischer Diskussionen sind und Fragen der beiden vorangehenden Typen aufwerfen. Typischerweise finden diese Diskussionen in einem gesellschaftlichen Kontext statt, der durch Konventionen, rechtliche Normierung und gesellschaftliche Institutionen bereits in einer Weise normativ vorstrukturiert ist, die besondere bereichsethische Beachtung verdient (s.u.). Festzuhalten ist vor allem zweierlei: Alle drei Fragen sind von praktischer Bedeutung, und ohne Antworten auf Fragen des ersten Typs sind auch Fragen des zweiten und dritten Typs nicht zu beantworten.

\section{5 | Ausgangspunkte ethischer Orientierung}

Begründete Entscheidungen: Wie also sind Fragen des ersten Typs zu beantworten? Auch Entscheidungen zwischen ethischen Theorien und Methoden sind vernünftig in dem Maße, in dem dafür gute Gründe angeführt werden können - etwas, das sich argumentativ für das jeweils Verteidigte 
ins Spiel bringen lässt. Soweit bestimmte Gründe ihrerseits nur innerhalb eines bestimmten Rahmens überzeugen können (aufgrund bestimmter Prämissen, auf Basis bestimmter Methoden oder innerhalb bestimmter theoretischer Vorannahmen), kann für diesen Rahmen erneut nach Gründen gefragt werden. Die Rede von der Theorie- und Methodenwahl muss in Bezug auf ethische Ansätze nicht im Sinne einer vernünftig nicht mehr einholbaren (`dezisionistischen`) Letztentscheidung verstanden werden, solange nicht schon gezeigt ist, dass einerseits keine weiteren Gründe mehr angeführt werden können (z. B. weil wir bei letzten Axiomen angekommen sind) und dass andererseits überhaupt sinnvolle, ernsthaft vertretbare Alternativen zu der zu verteidigenden Theorie oder Methode zur Verfügung stehen. Einige Gründe für oder gegen einzelne der oben vorgestellten moralphilosophischen Positionen sind im zweiten Teil des Lehrbuchs bereits skizziert worden. Das mag einen Eindruck davon vermitteln, auf welche Art Entscheidungen zwischen alternativen ethischen Ansätzen begründet werden könnten, und dadurch zur eigenen Urteilsbildung anregen. Eine erschöpfende Diskussion alternativer ethischer Theorien ist im Rahmen dieser Einführung jedoch weder möglich noch sinnvoll. Auch im Folgenden soll nicht der Versuch unternommen werden, einen eigenen umfassenden ethischen Ansatz $\mathrm{zu}$ entfalten und $\mathrm{zu}$ verteidigen. Wohl aber sollen einige Minimalannahmen über Möglichkeiten ethischer Rechtfertigung formuliert werden, die zwar philosophisch nicht gänzlich unstrittig sind (solche gibt es nicht), aber doch weitgehend theorieübergreifend akzeptiert werden.

\subsection{1 | Grundbedingungen der Rechtfertigbarkeit als Ausgangspunkt ethischer Orientierung}

Allgemeinheit von Gründen: Als Bestandteil einer minimalen Konsensbasis scheint zunächst folgende Annahme geeignet: `Gute Gründe müssen nachvollziehbar sein. Ihre Nachvollziehbarkeit setzt eine bestimmte Art von Verallgemeinerbarkeit voraus. Beispielsweise lässt sich in der Kombination der beiden Umstände, (P1) dass Sokrates ein Bürger Athens und (P2) dass Athen ein Stadt ist, ein nachvollziehbarer Grund dafür sehen, dass Sokrates die Gesetze Athens befolgen muss, wenn gilt, (P3) dass alle Bürger einer Stadt die Gesetze dieser Stadt befolgen müssen.

Prämisse 1: Sokrates ist ein Bürger Athens.

Prämisse 2: Athen ist eine Stadt.

Prämisse 3: Alle Bürger einer Stadt müssen deren Gesetze befolgen.

Konklusion: Sokrates muss die Gesetze Athens befolgen.

Brückenprinzipien Die verallgemeinernde Annahme, wonach alle Bürger einer Stadt die Gesetze dieser Stadt befolgen müssen, dient gewissermaßen als Brücke, über die sich von den konkreten Feststellungen P1 und P2 zu der Folgerung gelangen lässt, dass Sokrates die Gesetze Athens befolgen muss. Nachvollziehbare Wege von Gründen zu Folgerungen führen stets über vergleichbare Brücken der Verallgemeinerung. Das gilt ungeachtet der 
Tatsache, dass die in Anspruch genommenen Brückenprinzipien in Alltagsdiskussionen oft als selbstverständlich vorausgesetzt und daher nicht explizit gemacht werden. Wenn Leo seinem Wunsch, von seinen Eltern ein Eis zu bekommen, mit dem Hinweis Nachdruck verleiht, dass Schwester Lena auch eines bekommen hat, braucht er normalerweise nicht auf das Brückenprinzip »Alle Kinder sollten von ihren Eltern gleich behandelt werden!« aufmerksam zu machen - obwohl es mutmaßlich für die moralische Relevanz seines Hinweises entscheidend ist.

\section{Ethik des konkreten Anderen}

Auch phänomenologische Ethiken, die ethische Verpflichtungen in der Erfahrung eines 'konkreten Anderen grundgelegt sehen (Levinas 1987, 1989), müssen ihre Adressaten wohl über entsprechende Brücken der Verallgemeinerung führen, soweit sie denn bestimmte Adressaten von der Notwendigkeit bestimmter moralischer Reaktionen auf bestimmte Situationen überzeugen wollen. Denn die Position eines konkreten Anderen soll anscheinend von allen Menschen und gegenüber allen Menschen eingenommen werden können. Was es bedeutet, diese Position einzunehmen, kann sprachlich wiederum nicht anders denn in allgemeinen Begriffen umrissen werden. Soweit diese Deutung zutrifft, rekurriert offenbar auch die Ethik des `konkreten Anderen auf verallgemeinerbare Rollen und allgemeine Kriterien. Anderenfalls bliebe unklar, was überhaupt von wem und wem gegenüber normativ erwartet wird. Auch auf konkrete Personen, Erfahrungen, Haltungen oder Handlungsweisen zu zzeigen ist keine Alternative, wenn wir nicht schon irgendein allgemeines Vorwissen davon haben, worauf der zeigende Finger eigentlich deuten soll (z. B. auf eine Person und nicht auf ihren Kleiderstoff, auf das Muster dieses Stoffes oder auf das Pochen ihres Herzens; vgl. Wittgenstein, $P U, \S 33)$.

Gleichbehandlung und Supervenienzprinzip: Damit erscheinen bestimmte Formen von Egoismus unmittelbar unvernünftig. Willkürlich und daher unvernünftig wäre etwa die Behauptung, dass jemand deshalb bestimmte Rechte oder Pflichten hat, weil er nun einmal eine konkrete Person ist (z. B. dieser eine Sokrates aus Athen). Ebenso kann niemand vernünftigerweise gegenüber anderen Personen beanspruchen, dass ihre oder seine Interessen allein deshalb Vorrang vor denen anderer Personen haben sollen, weil sie nun einmal ihre oder seine eigenen sind. Potentiell vernünftige Ansprüche sind jedenfalls auf Gründe gestützt, die qua nachvollziehbare Gründe auf allgemeine Merkmale der betroffenen Personen, Handlungen oder Situationen bezogen werden können. Wenn Sokrates (oder einem Bürger Athens) vernünftigerweise Rechte oder Pflichten zuerkannt werden sollen, die anderen Personen (oder Bürgern anderer Städte) nicht zukommen, dann muss gezeigt werden, dass qualitative Unterschiede zwischen Sokrates und Anderen bzw. zwischen Bürgern Athens und Anderen vorliegen. Vernünftig können nur Ansprüche sein, die dem von Platon formulierten Prinzip folgen, Gleiches gleich und (nur) Ungleiches ungleich zu behandeln. Dieser Grundsatz folgt auch aus dem

Willkürlichkeit des Egoismus 
in der (Meta-)Ethik allgemein akzeptierten Supervenienzprinzip. Es besagt, dass moralische Eigenschaften über nicht-moralische bzw. natürliche Eigenschaften »supervenieren« und sich nur zugleich mit diesen ändern können.

\section{Verallgemeinerter Egoismus?}

Das Prinzip der Gleichbehandlung gleicher Fälle schließt nicht alle Formen des ethischen Egoismus unmittelbar aus. Vereinbar scheint es mit einem verallgemeinerten ethischen Egoismus, der besagt, dass jede Person ihren je eigenen Interessen stets Vorrang vor denen aller Anderen geben oder dass sie überhaupt nur die Maximierung der je eigenen Interessen anstreben sollte. Dieses Prinzip liefe allerdings auf einen Relativismus hinaus, in dem die abweichenden Prioritäten der verschiedenen Akteure unvereinbar nebeneinander (und oft gegeneinander) stehen. Würden wir das Prinzip des verallgemeinerten Egoismus uneingeschränkt akzeptieren, stünde kein gemeinsamer Beurteilungsstandpunkt zur Verfügung, von dem aus wir uns auf Prinzipien legitimer Kooperation einigen könnten. Damit bleibt unklar, wie sich für das Prinzip des verallgemeinerten Egoismus widerspruchsfrei argumentieren lässt: Soll auch noch die Akzeptanz des Egoismusprinzips unter dem Vorbehalt stehen, dass die Zustimmung zu diesem oder einem anderem Prinzip die Befriedigung der Privatinteressen der Zustimmenden maximiert? Dann würden wohl, je nach Situation und individueller Interessenlage, von verschiedenen Personen ganz unterschiedliche Prinzipien akzeptiert. Oder soll das Prinzip des verallgemeinerten Egoismus durch andere, nicht-egoistische Gründe gerechtfertigt werden? Dann wäre implizit zugestanden, dass wir uns in bei der Wahl der letzten ethischen Prinzipien gerade nicht einfach an interessenrelativen Privatgründen orientieren sollen, sondern an einer Art normativer Gründe, die wir teilen können.

Diskriminierung und Differenzblindheit
Relevanz von Unterschieden: Das Supervenienzprinzip ist konstitutiv für die Rechtfertigungspraxis und damit a priori wahr bzw. eine "Plattitüde" (Smith 1994, S. 22, 182). Die gleiche Behandlung qualitativ gleicher Fälle sichert aber für sich genommen noch keine Gerechtigkeit (vgl. auch zum Folgenden Mackie 1977, Kap. 4; Wimmer 1980). Denn sie schließt nicht unmittelbar aus, dass irrelevante Unterschiede zur Begründung von Ungleichbehandlung angeführt werden, wie es beispielsweise der Fall ist, wenn das Wahlrecht von der Zugehörigkeit zu einem bestimmten Geschlecht abhängig gemacht wird. In solchen und ähnlichen Fällen willkürlicher Ungleichbehandlung sprechen wir im pejorativen Sinn von Diskriminierung. Das formale Gleichbehandlungsprinzip schließt auch umgekehrt nicht unmittelbar aus, dass relevante Unterschiede, die tatsächlich für eine ungleiche Behandlung sprechen, ignoriert werden; etwa, wenn den besonderen Bedürfnissen, Kompetenz- oder Belastungsgrenzen von Kindern oder Personen mit physischen oder psychischen Einschränkungen nicht angemessen Rechnung getragen wird. Hier könnte man von Differenzblindheit sprechen.

Konsistenz: Allerdings ist mit der Suche nach verallgemeinerbaren 
Gründen ein Prozess in Gang gesetzt, der sich wiederum nicht wie Schopenhauers Fiaker einfach anhalten lässt. Denn der Anspruch, dass be-

Kritik inkonsistenter Positionen stimmte Unterschiede relevant für die Zuerkennung von Rechten oder Pflichten sind, kann seinerseits nur in den Maße als vernünftig gelten, als sich dafür wiederum überzeugende allgemeine Gründe anführen lassen. Dieses Erfordernis zieht Konsistenz- und Nachweispflichten nach sich, die sich in den sozialen Kämpfen gegen willkürliche Diskriminierungen einklagen lassen. Ein einschlägiges historisches Beispiel ist die Argumentation von John Jay, einem der >Gründerväter der Vereinigten Staaten. In einem Brief aus dem Jahr 1786 führt er aus, dass die Sklaverei mit den Idealen der amerikanischen Unabhängigkeitsbewegung unvereinbar sei. Wer sich in seinem Streben nach politischer Autonomie selbst auf Freiheitsrechte berufe, könne nicht zugleich Sklaverei dulden: »To contend for our own liberty, and to deny that blessing to others, involves an inconsistency not to be excused « (Jay 1833, S. 231). Die Stärke wie die Schwäche von Konsistenzargumenten liegt darin, dass sie lediglich auf Voraussetzungen rekurrieren, die auch die Gegenseite in Anschlag bringen. Jay hatte insofern leichtes Spiel, als seine Adressaten bereits auf universelle Menschenrechte pochen. Gleichwohl lassen sich Konsistenz- und Nachweispflichten auch schon gegen Aristoteles' Versuch einer Rechtfertigung der Sklaverei wenden. Seine Behauptung, es gebe "Sklaven von Natur", für die es besser sei, von anderen regiert zu werden, weil sie selbst $\mathrm{zu}$ unvernünftig seien, als dass sie für ihr eigenes Wohl sorgen könnten (siehe Kap.2.2), zwingt ihn nicht nur selbst zur Einsicht, dass es sich jedenfalls nicht bei allen Sklaven um »Sklaven von Natur« handelt (Aristoteles, Politik, 1255a2-b15). Sie macht auch die Legitimität der Sklaverei von einigen mehr als fragwürdigen empirischen Annahmen abhängig. Gerade die schönfärberische Beschreibung des Versklavtseins als im besten Interesse der Sklaven liegend und daher sogar der Freundschaft verwandt, stellt die Legitimität der Sklaverei unter eine Bedingung, der sie unmöglich genügen konnte.

Unparteilichkeit: Die vorangehenden Überlegungen sind bedeutsam für die Einordnung eines Leitmotivs, das in den verschiedenen in Teil II behandelten Theorien immer wieder angeklungen ist: das Ideal der Unparteilichkeit. Kriterien und Modelle wie die kulturübergreifend verbreitete Goldene Regel (siehe Kap. 6.2.1; vgl. Carson 2013), der unparteiische Beobachter der schottischen Sentimentalisten, das utilitaristische Nutzenprinzip, der Kategorische Imperativ oder der rawlssche Urzustand lassen sich als alternative Versuche verstehen, dieses Ideal in möglichst prägnanter Weise auszubuchstabieren. Die vorangehenden Überlegungen sprechen nun dafür, dass die für eine nachvollziehbare Rechtfertigung notwendigen Verallgemeinerungen bereits als solche eine Tendenz zur Unparteilichkeit bedingen; eine Tendenz, die sich, wie angedeutet, sogar gegen die Intentionen derjenigen durchsetzen kann, die willkürliche Praktiken zu rechtfertigen suchen. Es kann an dieser Stelle offen bleiben, inwieweit sich auf der Ebene individueller moralischer Lernprozesse ähnliche Tendenzen zur Verallgemeinerung und Erweiterung der Moralgemeinschaft feststellen lassen, wie die kognitivistische Moralpsychologie im Anschluss an Piaget und Kohlberg nachzuweisen sucht (Colby/
Kritik empirischer Behauptungen 
Unbegrenzte

Moralgemeinschaft

Zur Vertiefung
Kohlberg 2011; Zizek/Garz/Nowak 2015). Ebenso kann offen bleiben, ob es neben kognitiven Gründen für diese Tendenzen auch noch `naturalistischeく Erklärungen gibt, etwa auf evolutionstheoretischer Grundlage (zur Diskussion Kitcher 2011; Singer 2011).

Universalismus: Garantiert allein schon das Erfordernis, Prinzipien des Zusammenlebens und ihre Begründungen in allgemeiner Form zu formulieren, moralische Unparteilichkeit? Oder ist es möglich, dass auch allgemein formulierte Prinzipien, die aufgrund allgemein formulierter Gründe als gültig anerkannt werden, noch Parteilichkeit ausdrücken etwa weil diese Gründe ihrerseits nur aus der partikularen Perspektive bestimmter Personen oder Gruppen als stichhaltig oder relevant anerkannt werden? Hier lässt sich darauf hinweisen, dass sich auch noch für Antworten auf die Frage, ob die Legitimität von Handlungsprinzipien aus der Perspektive bestimmter Personen oder Gruppen oder vielmehr aus der universellen Perspektive aller Moralsubjekte beurteilt werden muss, nach Gründen fragen lässt. So könnten etwa - durchaus gute! - Gründe dafür sprechen, dass bestimmte praktische Festlegungen (z. B. bestimmte Vereinsregeln) nur innerhalb bestimmter Gemeinschaften (z. B. in dem jeweiligen Verein) erfolgen sollten - etwa soweit sich dort besondere normative Erwartungen, Rechte und Pflichten ergeben, die Außenstehende nicht betreffen. Die Beurteilung der Gründe für oder gegen eine solche (inhaltlich eingeschränkte) Entscheidungsautonomie kann aber nicht wiederum schon exklusiv derjenigen Gemeinschaft überlassen werden, deren Entscheidungsautonomie zuallererst gerechtfertigt werden soll. Denn dies liefe auf eine zirkuläre Selbstermächtigung hinaus. Sie würde sich strukturell nicht von der Willkür eines Solipsisten unterscheiden, der nur seine eigene normative Autorität anerkennt und damit jeden Anspruch auf Nachvollziehbarkeit aufgibt. Wenn wir Legitimität an die Möglichkeit einer vernünftigen Rechtfertigung koppeln, bleibt daher nur die Möglichkeit, die Gemeinschaft derjenigen, deren normatives Urteil zählt, als grundsätzlich unbegrenzt zu verstehen, also letztlich einen Universalismus hinsichtlich der moralischen Entscheidungsautorität zu akzeptieren.

\section{Universalismus der Wissenschaftspraxis}

Ein vergleichbarer Universalismus kennzeichnet die Rechtfertigungspraxis der Wissenschaften: Auch hier gibt es zwar Fachgesellschaften mit begrenzter Mitgliedschaft, formale Qualifikationsgrade, wissenschaftliche Hierarchien und Unterschiede im akademischen Status. Deren Rechtfertigbarkeit, genaue Ausgestaltung, Nutzen und Gefahren sind jedoch wiederum Gegenstand einer im Hinblick auf Teilnehmer/innen, Beiträge und Ergebnisse offenen Diskussion. Vor allem gilt: Die Gemeinschaft der Wissenschaftler/innen darf nicht willkürlich Argumente bestimmter Personen oder Gruppen von der Berücksichtigung im wissenschaftlichen Diskurs ausschließen und zielt auf Ergebnisse, die uneingeschränkt (für alle) zustimmungsfähig sind. Sie muss wissenschaftliche Behauptungen und Argumente grundsätzlich »ohne Ansehen der Person« beurteilen, allein anhand der Kriterien, die sich im bisherigen wissenschaftlichen Diskurs 
durchgesetzt haben und die ihrerseits für kritische Diskussionen und Weiterentwicklungen offen sind. Diese Formen von Universalismus und Unparteilichkeit sowie des Absehens vom unmittelbaren Eigeninteresse sind wesentlich für die Integrität der Wissenschaftspraxis (vgl. klassisch Merton 1973). Die Unterschiede zwischen wissenschaftlicher Erkenntnispraxis und praktischer Rechtfertigung wären genauer zu erläutern; jedenfalls ist die Praxis der Rechtfertigung und Kritik in beiden Bereichen an ähnliche Idealisierungen und Normen gebunden (siehe Kap. 11.3).

Idealisierungen und Fallibilismus: Die vorangehenden Überlegungen kommen mit Scanlons Annahme überein, dass nur Prinzipien legitim sind, die niemand vernünftigerweise zurückweisen könnte (Scanlon 1998). Diese Annahme buchstabiert die Bedeutung von Legitimität aus. Sie ist idealisierend, weil unter realen Handlungsbedingungen die Möglichkeiten, vernünftige Gründe und Gegengründe zu prüfen und die möglichst qualifizierte Zustimmung anderer einzuholen, stets begrenzt sind. Auch ist nicht auszuschließen, dass sich bestimmte Methoden und Standards dieser Prüfung weiterentwickeln, ebenso wie dies in Bezug auf wissenschaftliche Methoden und Standards der Fall ist. Daraus lässt sich folgern, dass konkrete Moralurteile grundsätzlich fallibel sind, sich also im Nachhinein als unberechtigt erweisen können - so, wie dies ja auch auf wissenschaftliche Erkenntnisse zutrifft. Gut begründete Positionen mögen sich im Licht späterer Einsichten als unhaltbar erweisen. Trotzdem müssen wir uns, solange wir uns um Rechtfertigung bemühen, zutrauen, richtige Antworten finden zu können. Denn es gibt schlicht keine vernünftige Alternative dazu, den besten jeweils verfügbaren Gründen zu folgen. Tatsächlich haben wir ja durchaus eine Vorstellung davon, was es konkret bedeutet, sich gemeinsam um Regelungen zu bemühen, die von allen nach gründlicher Prüfung der einschlägigen Argumente und in Anbetracht möglicher Alternativen zwanglos akzeptiert werden könnten. Vor allem haben wir ein recht klares Bild davon, was es bedeutet, sich nicht ernsthaft um eine vernünftige Einigung zu bemühen, sondern beispielsweise einen Teil der von praktischen Regelungen Betroffenen willkürlich auszugrenzen, Evidenzen zu ignorieren oder interessenabhängig $\mathrm{zu}$ verzerren oder überhaupt inkonsistent zu argumentieren. Auch ethischer Fortschritt verdankt sich vor allem der Elimination des NichtRechtfertigbaren.

Urteilssubjekte, Handlungssubjekte, Anspruchssubjekte: Der durch die vorigen Überlegungen gestützte Universalismus betrifft die moralische Handlungsgemeinschaft und die moralische Urteilsgemeinschaft. Alle, d. h. alle, die überhaupt imstande sind, sich an praktischen Prinzipien zu orientieren, sollen sich an solchen Prinzipien des Zusammenlebens orientieren, die von niemandem, d. h. von keinem moralisch urteilsfähigen Wesen, vernünftigerweise zurückgewiesen werden können. Zwei Fragen sind damit noch nicht beantwortet:

1. Wie weit erstreckt sich die moralische Anspruchsgemeinschaft - die Gemeinschaft derer, deren Wohl, Bedürfnisse, Präferenzen oder Interessen moralische Berücksichtigung verdienen? 
Anforderungen an die Form legitimer Prinzipien

Voraussetzungen moralischer Mitbestimmung
2. Inwieweit lassen sich aus den bisherigen Überlegungen Folgerungen hinsichtlich der Frage gewinnen, welchen Bedingungen legitime Prinzipien des Zusammenlebens genügen müssen, und inwieweit tragen sie zur Klärung substantieller moralischer Fragen bei?

Auf die erste Frage wird im Zusammenhang mit der Tier- und Umweltethik eingegangen (siehe Kap. 11.2). Dort wird für die Position argumentiert, dass alle Entitäten, denen wir sinnvollerweise ein seigenes` Wohl oder >eigene` Bedürfnisse, Präferenzen oder Interessen zuschreiben können, moralische Berücksichtigung verdienen - dass also die Grenzen der moralischen Anspruchsgemeinschaft weiter gezogen sind als die der moralischen Urteils- und Handlungsgemeinschaft. Die damit verteidigte Position ist in der Literatur allerdings strittig. Im Folgenden wird zunächst die zweite Frage diskutiert.

Caveat: Bereits gezeigt wurde, dass die Legitimität von Prinzipien des Zusammenlebens jedenfalls Folgendes voraussetzt:

1. die Allgemeinheit der Form dieser Prinzipien sowie der sie stützenden Argumente,

2. die Relevanz der eingeführten Unterscheidungen,

3. die Konsistenz von Rechtfertigungen,

4. die Haltbarkeit in Anspruch genommener empirischer Annahmen.

Diese Anforderungen an die Form legitimer Prinzipien und ihrer Rechtfertigung sind vergleichsweise unkontrovers. Offenbar lassen sie sich nicht leicht zurückweisen. Auf die folgenden Überlegungen trifft das zumindest nicht in demselben Maße zu. Sie betreffen die Frage, inwieweit sich aus den obigen Überlegungen auch schon Anforderungen an den Inhalt legitimer Prinzipien des Zusammenlebens ergeben. Für sie wird an dieser Stelle nur Plausibilität behauptet. Sie bedürften in jedem Fall einer ausführlicheren Erörterung und Begründung als sie an dieser Stelle zu leisten ist.

Wahrung der Deliberations- und Handlungsfähigkeit: Folgendes scheint zumindest plausibel: Wenn alle, die dazu imstande sind, allgemein zustimmungsfähige (bzw. von niemandem vernünftigerweise zurückweisbare) Normen einhalten sollen, dann sollten sie sich auch um Bedingungen bemühen, in denen sie

1. möglichst gut imstande sind, gemäß solcher Prinzipien zu handeln und

2. die allgemeine Zustimmungsfähigkeit von Prinzipien des Zusammenlebens auf möglichst vernünftige Weise prüfen können.

Das impliziert die Pflicht, alle verantwortungsfähigen Wesen als moralische Mit-Gesetzgeber/innen (Kant, GMS, S. 438) bzw. als "self-originating sources of valid claims " (Rawls 1980, S. 546) zu achten, denen ein »Recht auf Rechtfertigung " zukommt (Forst 2007; vgl. Darwall 2006) und deren Urteilsautonomie und Handlungsfähigkeit $\mathrm{zu}$ schützen und $\mathrm{zu}$ wahren sind. Plausibel ist es dann auch, eine generelle Mitverantwortung für soziale Institutionen zu postulieren, die individuelle Autonomie sichern und vernünftige gemeinsame Deliberation und Beschluss- 
fassung sowie planmäßiges individuelles und kollektives Handeln ermöglichen. In der Plausibilität dieser Überlegungen liegt zumindest eine Basis für die Etablierung von Grundrechten hinsichtlich individueller Selbstbestimmung und Selbstentfaltung, basaler Kommunikationsfreiheiten und der Mitwirkung in den das kollektive Handeln regulierenden Institutionen. Zweifellos lässt sich die genaue Ausgestaltung solcher Grundrechte nicht schon aus den Bedingungen vernünftiger Rechtfertigung deduzieren. Richtig ist jedoch auch, dass vernünftige Diskussionen die Anerkennung der normativen Autorität potentieller Teilnehmer/innen immer schon zur Voraussetzung haben (Habermas 1992; für eine kritische Rekonstruktion vgl. Brune 2010).

\subsection{2 | Faktischer Konsens oder Theoriekonvergenz als Ausgangspunkte ethischer Orientierung}

Moralische Gewissheiten: Mit den vorangehenden Überlegungen ist eine erste Möglichkeit skizziert, innerhalb des pluralistischen Ethikdiskurses Orientierung zu finden: im Ausgang von den Grundbedingungen praktischer Rechtfertigung überhaupt. Eine zweite Möglichkeit, die sich auch als Ergänzung denken lässt, liegt im Rekurs auf Überzeugungen, die faktisch nicht - ernsthaft - bestritten werden. Zwar lässt sich im philosophischen Seminar fast alles bezweifeln. Nicht jeder grundsätzlich mögliche Zweifel ist aber lebenspraktisch bedeutsam: Wenn vor unseren Augen jemand zusammenbricht, dürfen wir keine Zeit mit der Spekulation verlieren, ob es tatsächlich eine Außenwelt gibt oder ob nicht alle menschenähnlichen Wesen nur avancierte Roboter sind. Wir müssen versuchen, Hilfe zu leisten. Das Maß hinreichender Gewissheit ist kontextbezogen; und in vielen Kontexten, in denen Orientierung durch ethische Beratung oder im ethischen Dialog gesucht wird, teilen die Beteiligten wesentliche moralische Überzeugungen. Auf diesen Umstand stützen sich Ansätze wie der oben diskutierte Principlism, der common morality Ansatz aber auch viele andere Ansätze (beispielsweise der Pragmatismus oder Intuitionismus) durchaus zu Recht. Wer wollte ernsthaft, nicht nur im Sinne eines rein spekulativen paper doubt (Peirce 1931, Bd. 6, S. 498), bestreiten, dass körperliches Leiden etwas intrinsisch Schlechtes ist (Nagel 1986) oder dass Lüge, sinnlose Leidzufügung oder Grausamkeit prima facie verwerflich sind (Margalit 1996)? Von Aristoteles bis heute setzen ethische Begründungsbemühungen immer wieder bei solchen vergleichsweise unstrittigen Überzeugungen an (beim common sense, oder dem consensus gentium, bei geteilten moralischen Intuitionen oder considered judgments), um von dort aus Antworten auch auf weniger eindeutige Fragen zu entwickeln.

Theoriekonvergenz: In den gegenwärtigen Diskursen der bereichsspezifischen Ethik werden solche Versuche meist kohärentistisch interpretiert: Die genannten Überzeugungen werden dann als zentrale Ausgangs- und Bezugspunkte von Bemühungen zur Herstellung eines Überlegungsgleichgewichts verstanden (siehe Kap.7.2.2). Weil im Rahmen des Überlegungsgleichgewichts vortheoretische Überzeugungen und Theorieelemente wechselseitig abgeglichen werden, können nicht nur kon-

Ausgang vom hinreichend Unstrittigen 
krete, einzelfallbezogene moralische Überzeugungen, sondern auch >mittlere Prinzipien (siehe Abb.5.1) als Ausgangspunkte dienen. Zur Bekräftigung einer moralischen Überzeugung mag auch der Nachweis dienen, dass sie durch ein breites Spektrum prima facie plausibler Ethiktheorien nahegelegt wird. Allerdings kann weder der Ausgang von substantiellen moralischen Überzeugungen noch der Rekurs auf Theoriekonvergenz zuverlässig die Gültigkeit des Ergebnisses von Begründungsbemühungen verbürgen. Beispielsweise mag sich Konsens auf der Ebene mittlerer Prinzipien auflösen, sobald man versucht, diese auf konkrete Fälle zu beziehen oder gegeneinander abzuwägen. Das spricht aber nicht gegen solche Versuche: Ein Klärungsfortschritt liegt auch in der Erkenntnis oder genaueren Rekonstruktion von Dissensen, die ja womöglich in späteren Versuchen ausgeräumt werden können.

\subsubsection{Konditionales Argumentieren als Beitrag zur ethischen Orientierung}

Argumentation unter Vorbehalt
Einklammerung strittiger Voraussetzungen: In Hinsichten, in denen keine Konsensbasis in Anspruch genommen werden kann, kann sich philosophische Ethik oder ethische Beratung auf konditionale Argumentationen zurückziehen und strittige Voraussetzungen einklammern. Ethiker/ innen beschränken sich dann darauf, auszubuchstabieren, welche moralischen Verpflichtungen, Verbote oder Ratschläge sich für bestimmte Situationen oder Situationstypen ergeben, wenn man von diesen bestimmten ethischen Theorien oder Prinzipien ausgeht, wenn man die fraglichen Situationen auf diese bestimmte Weise interpretiert und wenn man dabei bestimmte empirische Annahmen akzeptiert. Mit solchen Aussagen beantworten Ethiker/innen quasi nicht selbst, was (unbedingt oder klugerweise) getan werden sollte. Sie leisten jedoch immerhin Beiträge zur Transparenz und Nachvollziehbarkeit der Urteilsbildung. In jedem Fall ist es im Rahmen ethischer Erörterung und Beratung geboten, dass nichttriviale Voraussetzungen der eigenen Argumentation, ganz gleich ob empirischen, begrifflichen, methodischen oder normativen Charakters, soweit wie möglich offengelegt werden.

Interdisziplinarität: Dass sich unter den in Anspruch genommenen Voraussetzungen auch Interpretationen und empirische Annahmen befinden, trifft für ethische Argumentationen generell zu (vgl. Scanlons Unterscheidung zwischen "reinen" und "gemischten" normativen Gründen; Scanlon 2014, S. 37 ff.). Schon abstrakte Normen wie »Du sollst nicht töten!« haben nicht-moralische Implikationen (beispielsweise, dass es sterbliche Lebewesen gibt). Insbesondere für Diskussionen der bereichsspezifischen Ethik, die sich regelmäßig mit neuartigen Handlungsfeldern befasst, gilt überdies, dass auch beurteilungsrelevante empirische Annahmen oft mehr oder weniger kontrovers sind. Daraus folgt, dass eine wissenschaftlich vertretbare bereichsethische Beratung am ehesten in der interdisziplinären Zusammenarbeit zwischen Moralphilosophie und den jeweils relevanten empirischen Disziplinen erfolgen kann (vgl. Düwell 2008, S. 5 ff.). 


\subsection{4 | Situationshermeneutik als Beitrag zur ethischen Orientierung}

Ethische Situationshermeneutik: Die Zusammenarbeit einzelner Disziplinen kann sich nicht darin erschöpfen, empirische und normative Elemente wie Bausteine zusammenzutragen, die nachträglich zusammengefügt werden. Das lässt sich wie folgt verdeutlichen: Ein wesentliches Element ethischen Urteilens über Situationen oder Situationstypen liegt in der interpretierenden Problemerschließung. Die moralisch relevanten Aspekte der Situation müssen - so scheint es - zunächst als solche wahrgenommen werden, ehe situationsethische Fragen gestellt, in empirische und normative Aspekte zerlegt und abgearbeitet werden können. In Wahrheit impliziert jedoch schon die vermeintlich nur vorbereitende Situationserschließung immer schon einen vorläufigen und eventuell nur impliziten Urteilsprozess, der normative und empirische Einschätzungen aufeinander bezieht. Nur im Licht normativer Vorannahmen heben sich bestimmte Beobachtungen vom belanglosen Hintergrund ab und werden bestimmte empirische Fragen bedeutsam; nur bestimmte Beobachtungen und empirische Vorannahmen berechtigen zu der Vermutung, dass ein bestimmter moralisch relevanter Fall vorliegen könnte. Mehr noch: Auch die Hoffnung, nach erfolgter Situationsinterpretation normative und empirische Fragen gesondert abarbeiten zu können, ist meist trügerisch. Denn in aller Regel bringen neue oder spezifischere Sacheinsichten wiederum weitere normative Fragen hervor und verlangen neue oder präzisierte normative Erkenntnisse für weiteren empirischen Klärungsbedarf. Die interdisziplinäre Zusammenarbeit, die für bereichsethische Diskurse kennzeichnend ist, vollzieht sich daher in der Regel als ein iterativer Prozess, in dem empirische und normativ-ethische Fragen und Erkenntnisse immer wieder aufeinander bezogen werden müssen. Dieser Dialog setzt auf beiden Seiten Übersetzungskompetenzen voraus.

Ethische Sensibilität: Zu betonen ist schließlich, dass ethische Situationshermeneutik nicht als rein kognitives Vermögen verstanden werden kann. Sie setzt eine moralische Sensibilität voraus, die ohne leibliche Erfahrung und das Vermögen der einfühlenden Imagination nicht denkbar ist. Man muss nicht selbst sam eigenen Leibe ‘ erfahren haben, was Demütigung, Schmerz oder Todesangst bedeuten, um die moralische Bedeutung einer Situation erschließen zu können, in der eine Gruppe Jugendlicher einen der ihren missbraucht (vgl. Musil 1906) oder sich anschickt, eine Katze zu verbrennen (das Beispiel stammt von Harman 1977, S. 4 ff.; vgl. dazu Elias 1976, Bd. 1, S. 375). Man muss dazu jedoch imstande sein, sich diese Erfahrungen zumindest ansatzweise zu vergegenwärtigen. Aufgrund der Rolle sinnlicher Wahrnehmungsqualitäten kann ethische Sensibilität auch nicht allein durch rationale Bemühungen hergestellt werden. Vielmehr muss sie auch durch die authentische Verarbeitung wacher Erfahrungen ästhetisch geschult werden. Die zentrale Rolle der Literatur für die moralische Bildung wird von den Vertreterinnen und Vertretern der narrativen Ethik zu Recht hervorgehoben.

Zusammenspiel von empirischen und normativen Annahmen 


\section{Siglenverzeichnis}

AA - Akademieausgabe (Kant 1902 ff.)

GMS - Grundlegung zur Metaphysik der Sitten (Kant, AA, IV, S. 385-463)

PU - Philosophische Untersuchungen (Wittgenstein 1984, Bd. 1, S. 225-618)

\section{Zitierte und weiterführende Literatur}

Aristoteles: Werke in deutscher Übersetzung. Berlin $1956 \mathrm{ff}$.

Beauchamp, Tom L./Childress James F.: Principles of Biomedical Ethics. New York/Oxford ${ }^{7} 2013$.

Birnbacher, Dieter: Analytische Einführung in die Ethik. Berlin/New York ${ }^{3} 2013$.

Brune, Jens P.: Moral und Recht: Zur Diskurstheorie des Rechts und der Demokratie von Jürgen Habermas. Freiburg i. Br. 2010.

Carson, Thomas: „Golden Rule«. In: LaFollette, Hugh (Hrsg.): The International Encyclopedia of Ethics. Malden MA 2013.

Clarke, Stanley G./Simpson, Evan: Anti-Theory in Ethics and Moral Conservatism. New York 1989.

Colby, Anne/Kohlberg, Lawrence: The Measurement of Moral Judgment. Cambridge 2011.

Darwall, Stephen L.: The Second-Person Standpoint: Morality, Respect, and Accountability. Cambridge MA/London 2006.

Descartes, René: Discours de la méthode - Von der Methode des richtigen Vernunftgebrauchs und der wissenschaftlichen Forschung. Hamburg ${ }^{2} 1997$ [EA anonym, Leyden 1637].

Düwell, Marcus: Bioethik: Methoden, Theorien und Bereiche. Stuttgart 2008.

Elias, Norbert: Über den Prozeß der Zivilisation. Zwei Bände. Frankfurt a. M. 1976.

Forst, Rainer: Das Recht auf Rechtfertigung: Elemente einer konstruktivistischen Theorie der Gerechtigkeit. Frankfurt a. M. 2007.

Fotion, Nick: Theory versus Anti-Theory in Ethics: A Misconceived Conflict. Oxford/New York 2014.

Gert, Bernard: Morality: Its Nature and Justification. New York/Oxford 1998.

Habermas, Jürgen: Theorie des kommunikativen Handelns. 2 Bände. Frankfurt a. M. 1981 .

Habermas, Jürgen: Moralbewusstsein und kommunikatives Handeln. Frankfurt a. M. 1983.

Habermas, Jürgen: Erläuterungen zur Diskursethik. Frankfurt a. M. 1991.

Habermas, Jürgen: Faktizität und Geltung: Beiträge zur Diskurstheorie des Rechts und des demokratischen Rechtsstaats. Frankfurt a. M. 1992.

Habermas, Jürgen: »Eine genealogische Betrachtung zum kognitiven Gehalt des Sollens«. In: Ders.: Die Einbeziehung des Anderen: Studien zur politischen Philosophie. Frankfurt a. M. 1996, S. 11-64.

Harman, Gilbert: The Nature of Morality: An Introduction to Ethics. Oxford 1977.

Hubig, Christoph: Die Kunst des Möglichen, Bd.2: Ethik der Technik als provisorische Moral. Bielefeld 2007.

Hubig, Christoph/Luckner, Andreas: »Klugheitsethik/Provisorische Moral«. In: Grunwald, Armin (Hrsg.): Handbuch Technikethik. Stuttgart 2013, S. 148-153.

Jay, William: The Life of John Jay: With Selections from His Correspondence. New York 1833.

Kant, Immanuel: Gesammelte Schriften. Hrsg. von der Königlich Preußischen Akademie der Wissenschaften. Berlin $1902 \mathrm{ff}$.

Kitcher, Philip: The Ethical Project. Cambridge/London 2011.

Levinas, Emmanuel: Totalität und Unendlichkeit: Versuch über die Exteriorität. Freiburg i. Br./München 1987.

Levinas, Emmanuel: Humanismus des anderen Menschen. Hamburg 1989.

Mackie, John L.: Ethics: Inventing Right and Wrong. Harmondsworth 1977.

Margalit, Avishai: The Decent Society. Cambridge 1996. 
Marshall, John: Descartes's Moral Theory. Ithaca/London 2018.

McMahon, Christopher: Reasonable Disagreement: A Theory of Political Morality. Cambridge/New York 2009.

Merton, Robert K.: The Sociology of Science: Theoretical and Empirical Investigations. Chicago 1973.

Mill, John St.: Collected Works. Toronto 1963 ff.

Multatuli: Ideën. Amsterdam 1877.

Musil, Robert: Die Verwirrungen des Zöglings Törleß. Wien/Leipzig 1906.

Nagel, Thomas: The View from Nowhere. Oxford/New York 1986.

Peirce, Charles S.: Collected Papers. Cambridge $1931 \mathrm{ff}$.

Perler, Dominik: René Descartes. München 1998.

Prichard, Harold A.: »Does Moral Philosophy Rest on a Mistake?« In: Mind 21/81 (1912), S. 21-37.

Rawls, John: Kantian Constructivism in Moral Theory. In: The Journal of Philosophy 77 (1980), S. 515-572.

Rawls, John: Political Liberalism. New York 1993.

Ricken, Friedo: Allgemeine Ethik. Stuttgart u. a. ${ }^{5} 2013$.

Ross, David: The Right and the Good [1930]. Oxford 2009.

Scanlon, Thomas M.: What We Owe to Each Other. Cambridge/London 1998.

Scanlon, Thomas M.: Being Realistic About Reasons. Oxford/New York 2014.

Schopenhauer, Arthur: Sämtliche Werke. Hrsg. von Arthur Hübscher. Mannheim ${ }^{4} 1988$.

Singer, Peter A.: The Expanding Circle: Ethics, Evolution, and Moral Progress. Princeton 2011.

Smith, Michael A.: The Moral Problem. Oxford/Cambridge 1994.

Weber, Max: Gesammelte Politische Schriften. Tübingen 1988.

Wimmer, Reiner: Universalisierung in der Ethik: Analyse, Kritik und Rekonstruktion ethischer Rationalitätsansprüche. Frankfurt a. M. 1980.

Wittgenstein, Ludwig: Werkausgabe in acht Bänden. Frankfurt a. M. 1984.

Zizek, Boris/Garz, Detlef/Nowak, Ewa (Hrsg.): Kohlberg Revisited. Rotterdam 2015.

Open Access Dieses Kapitel wird unter der Creative Commons Namensnennung 4.0 International Lizenz (http://creativecommons.org/licenses/by/4.0/deed.de) veröffentlicht, welche die Nutzung, Vervielfältigung, Bearbeitung, Verbreitung und Wiedergabe in jeglichem Medium und Format erlaubt, sofern Sie den/die ursprünglichen Autor(en) und die Quelle ordnungsgemäß nennen, einen Link zur Creative Commons Lizenz beifügen und angeben, ob Änderungen vorgenommen wurden.

Die in diesem Kapitel enthaltenen Bilder und sonstiges Drittmaterial unterliegen ebenfalls der genannten Creative Commons Lizenz, sofern sich aus der Abbildungslegende nichts anderes ergibt. Sofern das betreffende Material nicht unter der genannten Creative Commons Lizenz steht und die betreffende Handlung nicht nach gesetzlichen Vorschriften erlaubt ist, ist für die oben aufgeführten Weiterverwendungen des Materials die Einwilligung des jeweiligen Rechteinhabers einzuholen. 